Original article

\title{
Psychosocial contributing factors that affect mental well being in diabetic patients
}

\author{
Behshid Garrusi, Mohammad Reza Baneshi, Samaneh Moradi
}

Kerman University of Medical Sciences, Kerman, Iran

Received 19 November 2012, Revised 5 December 2012, Accepted 25 December 2012.

(C) 2012, Garrusi B., Baneshi M.R., Moradi S.

(C) 2012, Russian Open Medical Journal

\begin{abstract}
The rate of diabetes as a cause of disease burden has increased over time. Psychological well being could be considered as an important factor in improving of chronic disease management and decrease of mortality and morbidity of disease. Despite, increasing of Diabetes and its burden in Iran, there is a few researches about psychological aspects of treatment. The aim of this study was identifying of psychological contributing factors in determination of psychological well being in Iranian diabetic patients. Methods - This cross-sectional study was done in 350 diabetic patients that hospitalized for diabetes or related problems. The questionnaire's survey was assessed presence of depression and anxiety (Hospital Anxiety Depression Scale - HADS), family behaviors (Diabetes Specific Family Support - DSFS), quality of life (Iranian Diabetes QOL), self care (Diabetes Self-Care Activities Questionnaire) and mental health (General Health Questionnaire -GHQ). For analysis of data, we used Descriptive statistics, correlation and Two-way Analysis of Variance (ANOVA). P-value less 0.05 was considered to be significant. Results - About $2 / 3$ of participants had depression and anxiety. Anxiety, depression and non supportive behaviors had negative correlation with well being, quality of life and self care. Increasing of anxiety and depression was decreased the well being about $50 \%$ and $65 \%$, respectively. Being single would be decrease the mental well being about 2.5 times. Conclusion - The results of recent study support the recommendation to assess psychological well-being in patients with diabetes as one part of diabetes management.
\end{abstract}

Keywords: psychological well being, diabetes mellitus

Cite as Garrusi B, Baneshi MR, Moradi S. Psychosocial contributing factors that affect mental well being in diabetic patients. Russian Open Medical Journal 2013; 2: 0106.

Correspondence to Dr. Behshid Garrusi. Tel: +98-341-3224613. Fax: +98-341-3221671. E-mail \#1: bgarrusi@kmu.ac.ir. E-mail \#2: behshidgarrusi@gmail.com.

\section{Introduction}

Diabetes mellitus as a chronic disease that will be affect about 333 million in world by 2005 [1].The prevalence of diabetes is estimated about $5.5-7.7 \%$, in Iranian Adult Population [2, 3]. Diabetes increase the risk of long-term complications. The most important complications are cardiovascular disease, neuropathy, nephropathy, retinopathy (blindness). Such functional disabilities, treatment costs, dependency to others especially families, could be affect psychological well being in diabetic patients. The American Diabetes Association 'Standards for Medical Care' 2006 clearly states that emotional well-being is part of diabetes management, and recommends 'to incorporate psychological treatment into routine care rather than waiting for identification of a specific problem or deterioration in psychological status' [4].

Psychological well being have mutual effect in diabetic patients. Impairment in psychological well being is associated with insufficient glycemic control, lower rate of self control and increasing of diabetic complications [5]. On the other hand, the worsening of diabetes complications could be due to lower quality of life, depression and anxiety [6]. It is not surprising that association of psychological disorders and diabetes could be result to more severe of both complications such as higher work disabilities, insufficient treatment and lower regime or activity adherence [7].
Researchers are argue that psychosocial factors are the most important factors that could be intervene in well being of diabetic patients $[8,9]$.

Psychological well being is affected by many of psychiatric problems. Depression and anxiety have been associated with chronic disease such as diabetes. Anxiety and depression are common emotion in reaction to stressful issues, such as family and social problems. Moreover, some of factors such as illness may trigger anxiety. Symptoms of depression and anxiety may be fluctuating [5]. Depression and anxiety is associated with severe reduction of person's function and disease comorbidities [10].

One of the psychosocial factors that may be consider in diabetic patients' ell being is supportive environment that could be result to better metabolic management, therapeutic adherence and better quality of life $[11,12]$. One important aspect of social support is family support. Management of diabetes or its complication requires to change of lifestyles. These changing in lifestyles will be need helping from others especially near relatives. Some of researches are argue that not of all social or family supports may be useful [13].

Despite the number of studies regarding diabetes in Iran, considering to psychological aspects and related matters in these studies are low. Therefore, we decide to explore some if these 
factors in Iranian populations. Understanding these factors can help to better therapeutic approach and management of diabetes.

\section{Material and Methods}

This cross-sectional study was done in Kerman, the center of largest provinces in Iran as a doctorate thesis. The study was confirmed by the Ethics Committee at Kerman University of Medical Sciences.

\section{Participants}

Participants were included all of diabetic patients that hospitalized for diabetes or related problems from March to June 2012. Inclusion criteria were following:

i) age $>18$ years,

ii) hospitalization for diabetes type 2 and 3- desire for participation.

About 350 patients were participated in this study. Anonymous questionnaire was completed by the respondents after informed consent.

\section{Questionnaire}

This questionnaire were included below parts:

1 - Demographic variables such as gender, age, marital status (single, married, widowed), education (illiterate -elementary, high school, university), occupational status (unemployed, employed, housewife), economic status (fair-medium, good or excellent) and therapeutic agent (oral agents, insulin, both).

\section{2- Hospital Anxiety Depression Scale (HADS).}

This instrument was developed by Zigmond and Snaith in 1983. HADS has been developed for detection of depression and anxiety in hospitalized patients, but that would be suitable for using in the general population. Psychometric properties of this questionnaire in Persian was assessed that was acceptable. This scale have 14 items ( 7 items for each subscales) on a 4-point Likert scale (range 0-3), the total score is ranging from 0 to 21 [14]. HADS score was divided to three categories (for both depression and anxiety): 0-7 is normal, $8-11$ is borderline, and $>11$ represents clinical subjects.

3 - Diabetes Specific Family Support (DSFS).

This self reported likert type questionnaire was developed in 1986 by Schafer, that include 16 items, 9 items for positive or supportive behaviors and 7 items for negative or non supportive behaviors. This questionnaire is scored from 1 (none) to 5 (a lot of), the total score is ranging from 16 to 80 . Psychometric properties of this questionnaire in Persian was acceptable [15].

\section{4- Quality of life (Iranian Diabetes QOL).}

In this questionnaire there are 41 items regarding general ad specific aspects of quality of life. There were 13 items for assessing general QOL. The possible score was 40-160, higher scores indicating better QOL. This questionnaire had acceptable reliability and validity [16].

5-Summary of Diabetes Self-Care Activities (SDSCA) Questionnaire.

The questions ask about diabetes self-care activities during the past 7 days. The SDSCA is a 25 -item self-report measure of the frequency of performing diabetes self-care tasks over the preceding 7 days. This measure served as another index of patient self-care, to examine concurrent validity. Areas assessed include diet, exercise, glucose monitoring, medication taking, foot care, and smoking. Inter-item correlations ranged from $r=0.20$ to $r=0.76$ for four SDSCA subscales; 6-month test-retest reliability ranged from $r=0.00$ to $r=0.58$ across three studies [17].

\section{6 - General Health Questionnaire (GHQ-12).}

This is a screening instrument to detect psychiatric disorders in community settings and non-psychiatric clinical settings, such as primary care or general practice. This instrument was developed by Goldberg. Iranian version of the GHQ-12 has a good structural characteristic and is a reliable and valid instrument that can be used for measuring psychological well being in Iran [18].

\section{Statistical analysis}

Descriptive statistics were used to summarize the data, presentation of mean was mean $( \pm S D)$. The correlation between various variables was assessed by the Pearson correlation, Twoway Analysis of Variance (ANOVA) and last, linear regression analysis was performed to identify factors that influence mental health in a multi-factorial setting. All analyses were done using SPSS software. A P-value $<0.05$ was considered to be significant.

\section{Results}

About $72 \%$ of 351 participants were female. The mean age of subjects was $48.5( \pm 17.2)$ years. Demographic characteristics of participants were shown in Table 1.

Table 1. Demographic characteristics of participants

\begin{tabular}{|c|c|c|}
\hline Variables & $N(351)$ & $\%$ \\
\hline \multicolumn{3}{|l|}{ Gender } \\
\hline - Female & 253 & 72.1 \\
\hline - Male & 98 & 27.9 \\
\hline \multicolumn{3}{|l|}{ Marital status } \\
\hline - Married & 291 & 82.9 \\
\hline - Widowed & 42 & 12.0 \\
\hline - Single & 18 & 5.1 \\
\hline \multicolumn{3}{|l|}{ Work status } \\
\hline - Employee & 48 & 13.6 \\
\hline - Unemployed & 103 & 29.3 \\
\hline - Housewife & 200 & 56.9 \\
\hline \multicolumn{3}{|l|}{ Economic status } \\
\hline - Fair & 101 & 28.8 \\
\hline - Medium & 210 & 59.8 \\
\hline - Good or Excellent & 40 & 11.4 \\
\hline \multicolumn{3}{|l|}{ Living in } \\
\hline - Big cities & 206 & 58.7 \\
\hline - Village & 145 & 41.3 \\
\hline \multicolumn{3}{|l|}{ Kinds of treatment } \\
\hline - Oral agent & 101 & 28.8 \\
\hline - Insulin & 165 & 47.0 \\
\hline - Both & 60 & 17.1 \\
\hline - No treatment & 26 & 7.4 \\
\hline
\end{tabular}


Table 2. The mean score of Variables and Classification of psychiatric comorbidities

\begin{tabular}{lcc}
\hline \multicolumn{1}{c}{ Variables } & Mean & SD \\
\hline Depression & 9.48 & 2.55 \\
Anxiety & 9.39 & 4.53 \\
Family behaviors & & \\
• Supportive behaviors & 28.14 & 8.08 \\
• Non-Supportive behaviors & 11.86 & 4.98 \\
Quality of life & 56.88 & 1.61 \\
Self control & 25.06 & 13.29 \\
\hline \hline Classification of psychiatric co-morbidities & $N$ & $\%$ \\
\hline Anxiety & & \\
$\bullet$ Normal & 129 & 36.9 \\
$\bullet$ Borderline & 95 & 27.1 \\
$\bullet$ Clinical & 125 & 35.7 \\
Depression & & \\
$\bullet$ Normal & 69 & 19.7 \\
$\bullet$ Borderline & 168 & 48.0 \\
• Clinical & 111 & 31.7 \\
\hline
\end{tabular}

Table 3. The mean score of Variables based on gender

\begin{tabular}{|c|c|c|c|c|}
\hline Variables & Gender & Mean & $S D$ & $P$ value \\
\hline \multirow{2}{*}{$\begin{array}{l}\text { Mental Health } \\
\text { (GHQ) }\end{array}$} & Female & 15.07 & 7.06 & \multirow{2}{*}{ NS } \\
\hline & Male & 13.95 & 6.41 & \\
\hline \multirow{2}{*}{ Quality of life } & Female & 60.94 & 14.69 & \multirow{2}{*}{ NS } \\
\hline & Male & 60.61 & 13.17 & \\
\hline \multirow{2}{*}{ Anxiety } & Female & 9.79 & 4.51 & \multirow{2}{*}{0.025} \\
\hline & Male & 8.60 & 4.29 & \\
\hline \multirow{2}{*}{ Depression } & Female & 9.59 & 2.59 & \multirow{2}{*}{ NS } \\
\hline & Male & 9.74 & 2.38 & \\
\hline \multicolumn{5}{|c|}{ Family Behaviors } \\
\hline \multirow{2}{*}{ Supportive behaviors } & Female & 28.49 & 7.52 & \multirow{2}{*}{ NS } \\
\hline & Male & 30.15 & 18.17 & \\
\hline \multirow{2}{*}{ Non Supportive behaviors } & Female & 12.03 & 5.00 & \multirow{2}{*}{ NS } \\
\hline & Male & 11.97 & 4.98 & \\
\hline
\end{tabular}

Table 4. The mean score of Variables based on marital status

\begin{tabular}{|c|c|c|c|c|}
\hline Variables & Marital status & Mean & $S D$ & $P$ value \\
\hline \multirow{3}{*}{$\begin{array}{l}\text { Mental Health } \\
\text { (GHQ) }\end{array}$} & Single & 11.83 & 5.90 & \multirow{3}{*}{0.008} \\
\hline & Married & 14.56 & 6.87 & \\
\hline & Widowed & 17.40 & 6.80 & \\
\hline \multirow{3}{*}{ Quality of life } & Single & 60.37 & 14.41 & \multirow{3}{*}{0.004} \\
\hline & Married & 59.88 & 133.89 & \\
\hline & Widowed & 67.75 & 15.15 & \\
\hline \multirow{3}{*}{ Anxiety } & Single & 8.11 & 4.01 & \multirow{3}{*}{ NS } \\
\hline & Married & 9.32 & 4.47 & \\
\hline & Widowed & 10.92 & 4.46 & \\
\hline \multirow{3}{*}{ Depression } & Single & 9.50 & 3.12 & \multirow{3}{*}{ NS } \\
\hline & Married & 9.75 & 2.54 & \\
\hline & Widowed & 10.90 & 2.13 & \\
\hline \multicolumn{5}{|c|}{ Family Behaviors } \\
\hline \multirow{3}{*}{ Supportive behaviors } & Single & 26.38 & 8.35 & \multirow{3}{*}{ NS } \\
\hline & Married & 29.24 & 7.76 & \\
\hline & Widowed & 28.04 & 7.08 & \\
\hline \multirow{3}{*}{$\begin{array}{l}\text { Non Supportive } \\
\text { behaviors }\end{array}$} & Single & 11.33 & 3.91 & \multirow{3}{*}{0.002} \\
\hline & Married & 11.70 & 4.87 & \\
\hline & Widowed & 14.50 & 5.67 & \\
\hline
\end{tabular}

The mean score of variables and classification of psychiatric comorbidities were shown in Table 2.

The mean score of depression and anxiety were 9.5( \pm 2.6$)$ and $9.4( \pm 4.5)$, respectively. The mean score of GHQ was $15.0( \pm 7.7)$. Mean of supportive behaviors in family $\{28.1( \pm 8.1)\}$ was greater than non-supportive family behaviors $\{11.9( \pm 5.0)\}$. Except the score of Anxiety, there was no differences between other factors in both gender (anxiety score in male and was $8.6( \pm 4.3)$ and $9.8( \pm 4.5)$, respectively, $P=0.02)$. The mean score of some of predictive factors based on gender were shown in Table 3.

About $67 \%$ and $79 \%$ of diabetic patients had anxiety and depression, ordinary.

Table 4 is shown the differences of mean score of psychological variable based on marital status. The values of mental health, quality of life and non-supportive family behaviors were significantly different in various marital status.

There was negative correlation between anxiety, depression and non supportive family behaviors with mental health, quality of life and self care. Supportive family behaviors had negative correlation with depression and anxiety. The negative correlation was between non supportive behaviors and depression and anxiety (Table 5).

We used a multi factorial linear regression model to explore variables that influence mental health after adjusting for other variables. Results are given in Table 6. Anxiety, depression, family behaviors, education and marital status, were independently able to predict mental health. Marital status was significance, being single could be increase the chance of poor mental health about 2.5 times.

\section{Discussion}

The aim of medical care in chronic disease such as diabetes is not only physical treatment, but also to ameliorate of quality of life and decrease of mental health consequences. Some of researches were focused on gender differences in health consequences of disease. These researches, often reported lower quality of life and mental health in females [19]. This health consequences were in different age groups [20]. Despite of previous results, in recent study, there was no gender differences in mental health status. Except of biological factors that could be result to gender differences in reaction to disease, this difference was probably due to the effects of social factors [12].

The result of this study showed that self care in diabetes was affected negatively by depression and anxiety. Despite, prevalence of depression and anxiety in both gender is different for example women have twice the risk for most anxiety disorders as men [10, 20], but in this study among psychosocial variables, only anxiety is higher in women than men. In traditional Iranian families, the men are breadwinner and other family responsibilities such as child rearing and housekeeping duties, are expected from women. Constraints arising from diabetes such as specific regimen, taking medication, regular blood control and complication of disease could be induce higher stress in women.

Also, depression in diabetic patients are present about twice of general population [21]. Association between depression and diabetes could be affect the course of disease, health consequences, quality of life and even mortality [21]. Regression results showed both anxiety and depression will be increase chance of poor mental health. 
Clinical Psychology

Table 5. Correlation between psychosocial variables

\begin{tabular}{|c|c|c|c|c|c|c|c|}
\hline & Mental Health & Quality of life & Self care & Anxiety & Depression & $\begin{array}{c}\text { Supportive } \\
\text { family } \\
\text { behaviors }\end{array}$ & $\begin{array}{c}\text { Non- } \\
\text { supportive } \\
\text { family } \\
\text { behaviors }\end{array}$ \\
\hline Mental Health & 1 & & & & & & \\
\hline Quality of life & $0.460 * *$ & 1 & & & & & \\
\hline Self care & $0.113^{*}$ & 0.011 & 1 & & & & \\
\hline Anxiety & $-0.51 * *$ & $-0.450 * *$ & -0.027 & 1 & & & \\
\hline Depression & $-0.476 * *$ & $-0.377 * *$ & -0.089 & $0.422 * *$ & 1 & & \\
\hline Supportive family behaviors & 0.137 & 0.430 & $0.184 * *$ & -0.022 & -0.064 & 1 & \\
\hline Non-supportive family behaviors & $-0.233^{*} *$ & $-0.254 * *$ & -0.005 & 0.106 & 0.026 & -0.025 & 1 \\
\hline
\end{tabular}

$* \mathrm{P}>0.05, * * \mathrm{P}>0.01$

Table 6. Regression analysis of factors that influence mental well being in diabetic patients

\begin{tabular}{lll}
\hline & Beta & $S D$ \\
\hline Anxiety & -0.530 & 0.075 \\
Depression & -0.657 & 0.126 \\
Supportive family behaviors & 0.118 & 0.036 \\
Non-supportive family behaviors & -0.219 & 0.058 \\
Marital status & -2.559 & 1.220 \\
\hline
\end{tabular}

Another factors that could be affect the mental health was supportive behaviors from family. Some of behaviors from relatives or family could be harmful or negative. Cole found that negative family interactions may be induce lower daily self care and therapeutic compliance in diabetic patients [22]. Lake of family support or negative family support such as nagging about disease or treatment could be associate with poor glucose control [23]. Emotional well being and decrease of psychiatric co morbidities such as depression or anxiety would be decrease patients' distress, strain and increase of owns confidence. Self confidence could be resulting to better self care, quality of life and relation of family. Family behaviors such as communication, life styles and dynamic of family interaction could be influence of health out come such as depression, anxiety and perception of quality of life $[22,24]$.

As above mentioned, social network will be decrease adverse health consequences of chronic disease [22]. Marriage and marital life are considered as one of important social network. Some of researches are argue that more prolonged survival times and better health outcomes are associated with marriage [25]. Some of studies are focused on marital satisfaction as a predictor of poor metabolic control and elation of HbA1c [24]. In Iran, family is considered the most important and basic social group, therefore the role of family must be intentioned seriously. Except marriage, other demographic variables even duration of disease had no effect on well being.

The results of this study were consistent with findings regarding of researches that focused on relation between good socio-emotional support and improving of mental health wellbeing.

\section{Limitations}

For assessment of psychosocial predictive factors in chronic disease, studying the micro-cultures of a society may enhance our understanding of the social attitude towards disease, management and consequences.

\section{Conclusion}

Despite, mutual effects of mental well being and diabetes, attention to family dynamic and psychiatric co-morbidities especially anxiety and depression must be seriously considered. For obtaining of better results, it is expected, psychosocial factors are mentioned in diabetes management programs.

\section{Acknowledgment}

This research was financially supported by Neurosciences Research Center of Kerman University Medical Sciences as doctorate thesis. The authors sincerely thank Dr. Toobert and Dr. Alavi for giving us the opportunity of using questionnaires and their cooperation. Also we thank Dr. Neuzar Nakhaee for his guidance and cooperation.

Conflict of interest: none declared.

\section{Reference}

1. World Health Organization (WHO), 2010. Url: www.who.int/diabetes/ facts/world_figures/en.

2. Larijani B, Zahedi F. Epidemiology of diabetes mellitus in Iran. Journal of Diabetes and Metabolic Disorders 2002; 1: 7.

3. Esteghamati A, Gouya MM, Abbasi M, Delavari A, Alikhani S, Alaedini F, et al. Prevalence of diabetes and impaired fasting glucose in the adult population of Iran: National Survey of Risk Factors for NonCommunicable Diseases of Iran. Diabetes Care 2008; 31(1): 96-8. (PMID: 17921357)

4. American Diabetes Association. Standards of Medical Care in Diabetes - 2012. Diabetes Care 2005; 28(supp 1): S11-S63. (doi: 10.2337/dc12s011)

5. Engum A. The role of depression and anxiety in onset of diabetes in a large population-based study. I Psychosom Res 2007; 62: 31-38. (PMID: 17188118)

6. Grigsby $A B$, Anderson RJ, Freedland KE, Clouse RE, Lustman PJ. Prevalence of anxiety in adults with diabetes: a systematic review. $J$ Psychosom Res 2002; 53: 1053-60. (PMCID: PMC2782980)

7. National Alliance on Mental Illness. Url: www.nami.org.

8. Van Dam HA, van der Horst FG, Knoops L, Ryckman RM, Crebolder HF, van den Borne BH. Social support in diabetes: a systematic review of 
controlled intervention studies. Patient Educ Couns 2005; 59(1): 1-12. (doi: 10.1016/j.pec.2004.11.001) (PMID: 16198213)

9. Heeran C, Young-Ho K, II-Ho K, Sung-II C. Explaining gender differences in ill-health in South Korea: The roles of socio-structural, psychosocial, and behavioral factors. Soc Sci Med 2008; 67(6): 988-1001. (PMID: 18632197) (doi: 10.1016/j.socscimed.2008.05.034)

10. Kroenke K, Spitzer RL, Williams JB, Monahan PO, Löwe B. Anxiety disorders in primary care: prevalence, impairment, comorbidity, and detection. Ann Intern Med 2007; 146(5): 317-25. (doi: 10.3410/f.1088709.542826)

11. Spezia Faulkner M, Chang L. Family Influence on Self-Care, Quality of Life, and Metabolic Control in School-Age Children and Adolescents with Type 1 Diabetes. J Pediatr Nurs 2007; 22(1): 59-68. (PMID: 17234498)

12. Davis WK, Hess GE, Van Harrison R, Hiss RG. Psychosocial correlatesof survival in diabetes. Diabetes Care 1988; 11(7): 538-45. (PMID: 3203570)

13. Haynes RB. Improving patient adherence: state of the art, with a special focus on medication taking for cardiovascular disorders. In: Compliance in Health Care and Research. Burke LE, Ockene IS, eds. New York, NY: Futura Publishing Co Inc; 2001: 3-21.

14. Montazeri A, Harirchi AM, Shariati M, Garmaroudi GH, Ebadi M, Fateh A. The 12-item General Health Questionnaire (GHQ-12): translation and validation study of the Iranian version. Health Qual Life Outcomes 2003; 1: 66. (doi: 10.1186/1477-7525-1-66)

15. Morowati-Sharifabad MA, Rouhani Tonekaboni N, Baghianimoghadam $\mathrm{MH}$. Predictors of Self-Care Behaviors among Diabetic Patients Referred to Yazd Diabetes Research Centre Based on Extended Health Belief Model. The Journal Tabibe Shargh 2007; 9(4): 275-84. [Article in Persian]

16. Alavi NM, Ghofranipour F. Developing a culturally valid and reliable quality of life questionnaire for diabetes mellitus. East Mediterr Health J 2007; 13(1): 177. (PMID: 17546920)

17. Toobert DJ, Hampson SE, Glasgow RE. The summary of diabetes selfcare activities measure: results from 7 studies and a revised scale. Diabetes Care 2000; 23: 943-50. (PMID: 10895844)

18. Yaghubi H, Karimi M, Omidi A, Barooti E, Abedi M. Validity and factor structure of the General Health Questionnaire (GHQ-12) in university students. Journal of Behavioral Sciences 2012; 6(2): 15-6.

19. Unden A, Elofsson S, Andrasson A, Hillered E, Eriksson I, Brismar K. Gender Differences in Self-Rated Health, Quality of Life, Quality of Care, and Metabolic Control in Patients with Diabetes. Gend Med 2008; 5(2): 160-80. (doi: 10.1016/j.genm.2008.05.003) (PMID: 18573483)

20. Räty LKA, Larsson G, Söderfeldt BA, Wilde Larsson BM. Psychosocial aspects of health in adolescence: the influence of gender, and general self-concept. J Adolesc Health 2005; 36(6): 530.e21-530.e28. (doi: 10.1016/j.jadohealth.2004.10.006)

21. Gonzalez JS, Peyrot M, McCarl M, Collins EM, Mimiaga MJ, Safren SA. Depression and Diabetes Treatment Non adherence: A Meta-Analysis. Diabetes Care 2008; 31(12): 2398-403 (PMID: 19033420).

22. Cole I, Chesla CA. Interventions for the family with diabetes. Nurs Clin North Am 2006; 41(4): 625-39. (doi: 10.1016/j.cnur.2006.07.001)

23. Jones RA, Utz SW, Williams IC, Hinton I, Alexander G, Moore C, et al. Family interactions among African Americans diagnosed with type 2 diabetes. The Diabetes Educ 2008; 34(2): 318-26. (PMID: 18375781)

24. Hanson CL, Henggeler SW, Harris MA, Burghen GA, Moore M. Family system variables and the health status of adolescents with insulindependent diabetes mellitus. Health Psychol 1989; 8(2): 239-53. (PMID: 2737175)

25. Johnson NJ, Backlund E, Sorlie PD, Loveless CA. Marital status and mortality: the National Longitudinal mortality study. Ann Epidemiol 2000; 10: 224-38. (PMID: 10854957)
Authors:

Behshid Garrusi - MD, Associate Professor of psychiatry, Neuroscience research center, Kerman University of Medical Sciences, Kerman, Iran;

Mohammad Reza Baneshi - PhD, Assistant Professor of Statistics, Research Center for Modeling in Health, Institute of Futures Studies in Health, Kerman University of Medical Sciences, Kerman, Iran;

Samaneh Moradi - MD, Student Research Center, Kerman University of Medical Sciences, Kerman, Iran. 